\title{
Specified Object Tracking Problem in an Environment of Multiple Moving Objects
}

\author{
Seung-Min Park, Junheong Park, Hyung-Bok Kim and Kwee-Bo Sim* \\ School of Electrical and Electronics Engineering, ChungAng University
}

\begin{abstract}
Video based object tracking normally deals with non-stationary image streams that change over time. Robust and real time moving object tracking is considered to be a problematic issue in computer vision. Multiple object tracking has many practical applications in scene analysis for automated surveillance. In this paper, we introduce a specified object tracking based particle filter used in an environment of multiple moving objects. A differential image region based tracking method for the detection of multiple moving objects is used. In order to ensure accurate object detection in an unconstrained environment, a background image update method is used. In addition, there exist problems in tracking a particular object through a video sequence, which cannot rely only on image processing techniques. For this, a probabilistic framework is used. Our proposed particle filter has been proved to be robust in dealing with nonlinear and non-Gaussian problems. The particle filter provides a robust object tracking framework under ambiguity conditions and greatly improves the estimation accuracy for complicated tracking problems.
\end{abstract}

Key Words : Object tracking, particle filter, moving object, background image update, region-based tracking

\section{Introduction}

Much research is carried out in robotics concerning the detection of moving objects. Multiple Object Tracking, or MOT, is an experimental technique used to study how our visual system tracks multiple moving objects. Multiple objects tracking in unconstrained environment has many practical applications in scene analysis for automated surveillance, such as the detection of suspicious moving objects or the monitoring of an industrial production. If we can track a particularly selected object in an environment of multiple moving objects, then there will be a variety of applications. In this paper, we introduce a specified object tracking with particle filter in an environment of multiple moving objects.

When tracking, we need to analyze video sequences to track object in each frame. There are many tracking methods which fall into four categories in general: region-based tracking [1], model-based tracking [2], contour-based tracking [3] and feature-based tracking [4].

In this paper, we use a differential image of region-based tracking method for the detection of multiple moving objects. There are two methods which can be used to obtain a differential image between frames. One way is to use a background image and other one is to use a successive frames. But the latter does not appear an object when there is no movement of an object. So we use a background image to obtain a differential image. In order to ensure accurate object

Manuscript received Jan. 22, 2011; revised May. 18, 2011;

* Corresponding author

This work was supported by Mid-career Researcher Program through NRF grant funded by the Korean MEST(No. 20100029226). detection in unconstrained environment, we also use a method of background image update. When multiple objects were detected by using a differential image, we select particular object. And then selected object is tracked.

During tracking, the tracked object can be located by maximizing the similarity between a reference window and a candidate window. The maximum can be realized through either a deterministic way or a stochastic way.

Deterministic methods localize the tracked object in each frame by iteratively searching for a region which maximizes the similarity between this region and the target window. Comaniciu et al. [5] employed the Mean Shift algorithm for object tracking. These methods are computationally efficient. However, these methods may converge to a local maximum. They are sensitive to background distraction, clutter, occlusions, and quick moving objects.

Stochastic methods [6] however are able to maintain multiple hypotheses in the state space which can achieve more robustness to the local maximum. Kalman Filter can be applied to the linear-Gaussian estimation problem. The extended Kalman Filter (EKF) is the most popular approach to recursive nonlinear-Gaussian estimation. The particle filter [7], also known as sequential Monte Carlo methods, has been proved to be a robust algorithm to deal with the nonlinear, non-Gaussian problems.

In this paper, the particle filter provides a robust object tracking framework under ambiguity conditions and greatly improved estimation accuracy for complicated tracking problems. Particle filter have been widely introduced as a powerful and flexible tool. Particle filter provides an accurate estimate with low error variance, which is critical in object motion tracking.

This article is organized as follows. In Section 2 our system is explained. Section 3 describes background image update 
method. In Section 4, the principles of particle filters are briefly reminded. In Section 5, results are presented and analyzed. In this final chapter, we give the main conclusions of this paper and also briefly focus on some topics for future research

\section{System Overview}

The purpose of our work is to track particular object from an environment of multiple moving objects

Figure 1 shows a flow chart of system for multiple object detection and particular object tracking. Images obtained from the camera undergo Histogram Equalization preprocessing operation to reduce the effect of illumination change.

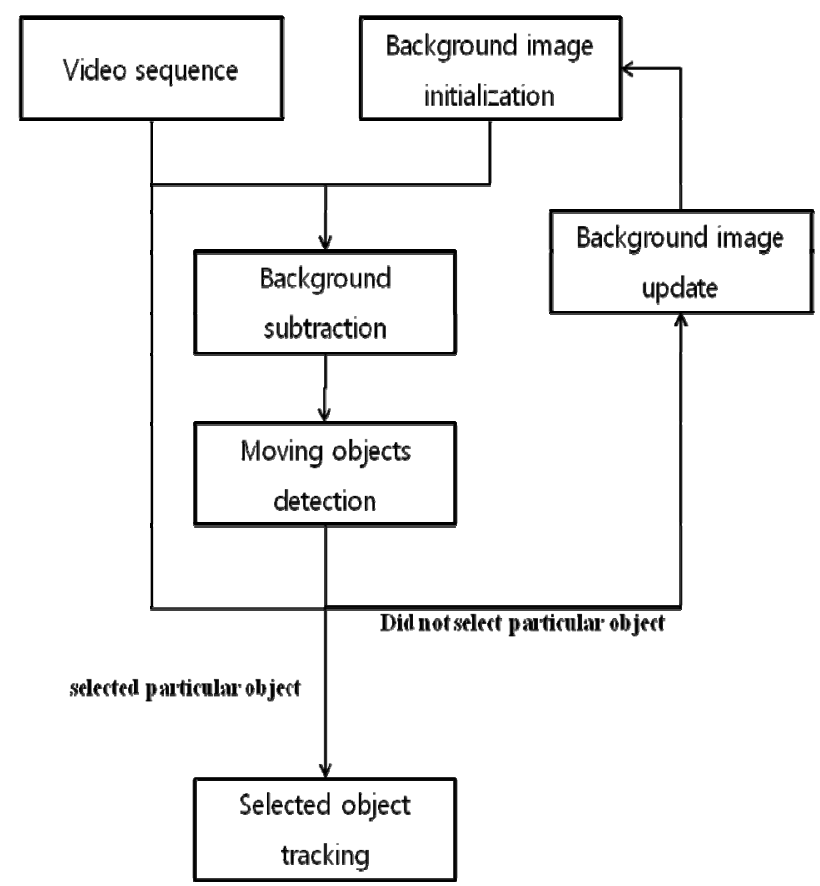

Fig. 1. A flow chart of System.

The initial background image is estimated as mean of frames obtained for a certain time. The mean background image will effectively remove camera noise and some casual moving articles appearing in the scene for short periods of time. Also background image is updated in every frame to ensure accurate moving object detection in unconstrained environment.

When multiple objects were detected by using a differential image, we select particular object. There are difficulties related to particular object tracking, such as erratic object motion, cluttered background and other moving objects. It can't rely only on image processing techniques. Thus we solved these problems using a probabilistic framework. Particle filter has been proved to be a robust algorithm to deal with the nonlinear, non-Gaussian problems. Particle filter provides a robust tracking framework and an accurate estimate with low error variance, which is critical in object tracking.

\section{Background Image Update}

In order to ensure accurate object detection in unconstrained environment, we use a method of background image update. This method uses a detected objects area of current frame and old background image to create a new background image in every frame. New background image is obtained by copying detected objects area of old background image into detected objects area of current frame. Figure 2 shows the process of a background image update.

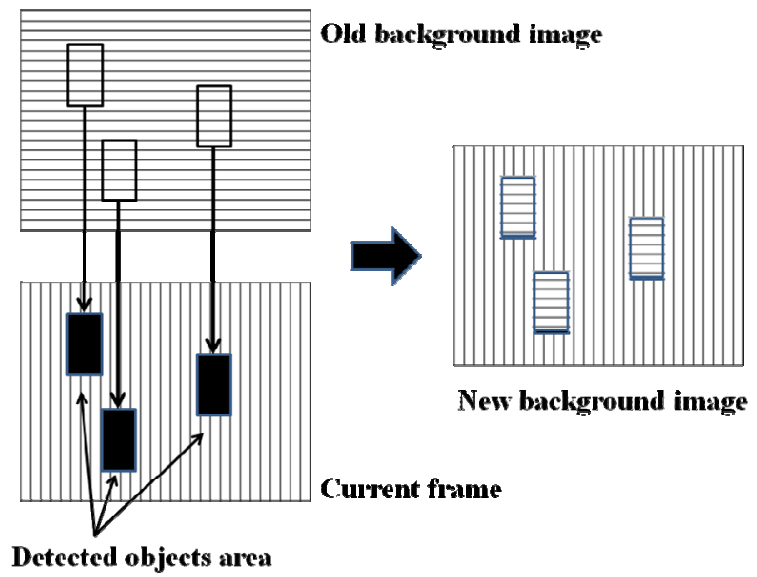

Fig. 2. The process of a background image update.

Step 1) Background image initialization

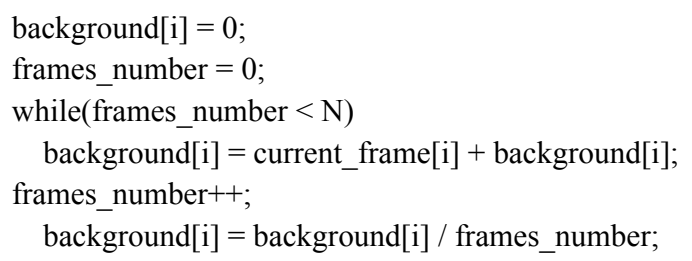

Step 2) Background subtraction

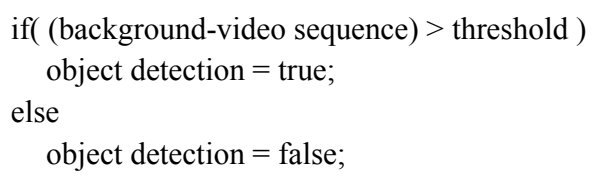

Step 3) Background image update

$$
\begin{aligned}
& \text { if(object detection) } \\
& \text { new_background }=\text { figure } 2 \text { method; } \\
& \text { else } \\
& \quad \text { new_background }=\text { video sequence; }
\end{aligned}
$$

\section{Particle Filter}

In this paper, Particle Filter is used to realize the specified object tracking. 
Particle filtering is a Monte Carlo approximation to the optimal Bayesian filter, which monitors the posterior probability of a first-order Markov process through the following formula:

$$
p\left(x_{t} \mid y_{1: t}\right)=\alpha p\left(y_{t} \mid x_{t}\right) \int_{x_{t-1}} p\left(x_{t} \mid x_{t-1}\right) p\left(x_{t-1} \mid y_{1: t-1}\right) .
$$

where, $\mathrm{xt}$ is the process state at time $\mathrm{t}, \mathrm{yt}$ is the observation, $\mathrm{y} 1: t$ is all of the observations through time $\mathrm{t}, \mathrm{p}(\mathrm{xt} \mid \mathrm{xt}-1)$ is the process dynamical distribution, $\mathrm{p}(\mathrm{yt} \mid \mathrm{xt})$ is the observation likelihood distribution, and $\alpha$ is a normalizing factor.

The integral in (1) does not have a closed form solution, except in the most basic cases, so particle filters are used to approximate (1) using a set of weighted samples $\left\{\mathrm{x}_{\mathrm{t}}^{\left({ }^{(}\right)}\right.$,

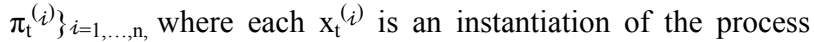
state, known as a particle, and the $\pi_{\mathrm{t}}^{(i)}$,s are the corresponding particle weights. Under this representation, the approximation to the Bayesian filtering equation (1) is

$$
p\left(x_{t} \mid y_{1: t}\right) \approx \alpha p\left(y_{t} \mid x_{t}\right) \sum_{i=1}^{n} \pi_{t-1}^{(i)} p\left(x_{t}^{(i)} \mid x_{t-1}^{(i)}\right) .
$$

To implement a standard particle filter, one must choose a state representation $x_{t}$, which, in the case of object racking might include object locations, scales, etc. In addition, one must design three distributions: the process dynamical distribution, $p\left(\mathrm{x}_{\mathrm{t}}^{(i)} \mid \mathrm{x}_{\mathrm{t}-1}{ }^{(i)}\right)$, which, in object tracking, describes how objects move between time steps; the proposal distribution, $\left(\mathrm{x}_{\mathrm{t}}{ }^{\left({ }^{i}\right)} \mid \mathrm{x}_{0: \mathrm{t}-1}{ }^{(i)}, \mathrm{y}_{1: \mathrm{t}}\right)$, which is sampled at each time step to update the particle distribution; and the observation likelihood distribution, $p\left(\mathrm{y}_{\mathrm{t}} \mid \mathrm{x}_{\mathrm{t}}{ }^{(i)}\right)$, which in tracking, describes how objects appear within the video data, $\mathrm{y}_{\mathrm{t}}$.

At each time step, given the previous particle set $\left\{\mathrm{x}_{\mathrm{t}-1}{ }^{(}\right), \pi_{\mathrm{t}}$ $\left.{ }_{1}^{(i)}\right\}$, a basic sequential importance resampling particle filter updates the particles as follows:

1. Sample $n$ particles $\mathrm{x}_{\mathrm{t}-1}{ }^{(i)}$ with replacement from current particle set according to probabilities $\pi_{\mathrm{t}-1}{ }^{(i)}$.

2. Generate an updated particle set by sampling from the proposal distribution, $\mathrm{x}_{\mathrm{t}}^{\left({ }^{(}\right)} \sim \mathrm{q}\left(\mathrm{x}_{\mathrm{t}}{ }^{(i)} \mid \mathrm{x}_{0: \mathrm{t}-1}{ }^{(i)}, \mathrm{y}_{1: \mathrm{t}}\right)$.

3. Reweight each particle according to the following formula and normalize so that the $\pi_{\mathrm{t}}^{(i)}$ sum to 1 :

$$
\pi_{t}^{(i)}=\frac{p\left(y_{t} \mid x_{t}^{(i)}\right) p\left(x_{t}^{(i)} \mid x_{t-1}^{(i)}\right)}{q\left(x_{t}^{(i)} \mid x_{0: t-1}^{(i)}, y_{1: t}\right)}
$$

In tracking and many other applications, it is typical to estimate the process state at each time step as the sample mean of the particles $\hat{E}\left[X_{t}\right]=\sum_{i=1}^{n} \pi_{t}^{(i)} X_{t}^{(i)}$

Follow table 1 is summary that particle filter execute iterative steps. And Figure 3 is that particle tilter describes iteration at once step.
Table 1. An iteration step of the particle filter

The given set of particle samples at initial step $\left\{\mathrm{X}_{\mathrm{t}-1}{ }^{(i)}, \pi_{\mathrm{t}-}\right.$ $\left.{ }_{1}^{(i)}\right\}$ help to gain the set of particle sample $\left\{X_{t}^{(i)}, \pi_{t}^{(i)}\right\}, t=$ $1,2, \ldots, \mathrm{N}$, Weight $\pi_{0}^{(\mathrm{n})}=1 \mathrm{~N}$ and cumulative distribution $\mathrm{c}_{0}{ }^{(\mathrm{n})}=1 / \mathrm{N}$ must be initialization.

1. Select the sample $\mathrm{s}_{\mathrm{t}}^{(\mathrm{n})}$ using follow methods.

- Generate the distributed random values uniformly.

- Find the smallest $\mathrm{j}$ of $\mathrm{c}_{\mathrm{t}-1}{ }^{(\mathrm{j})} \geq \mathrm{r}$.

- Define the $\mathrm{s}_{\mathrm{t}}^{(i)}=\mathrm{X}_{\mathrm{t}-1}{ }^{(i)}$

2. Predict by sampling for obtain to $s_{t}(i)$.

$$
\mathrm{P}\left(\mathrm{X}_{\mathrm{t}} \mid \mathrm{X}_{\mathrm{t}-1}=\mathrm{s}_{\mathrm{t}}{ }^{(i)}\right)
$$

3. Update the weight after measure feature parameter of

object.
It configure of become $\sum^{N} \pi_{t}^{(n)}=1$ and calculate the histogram distribution $\mathrm{c}_{\mathrm{t}}^{\left({ }^{(}\right)}$, upidate the set $\left\{\mathrm{X}_{\mathrm{t}}^{\left({ }^{(}\right)}, \pi_{\mathrm{t}}^{(i)}\right\}$

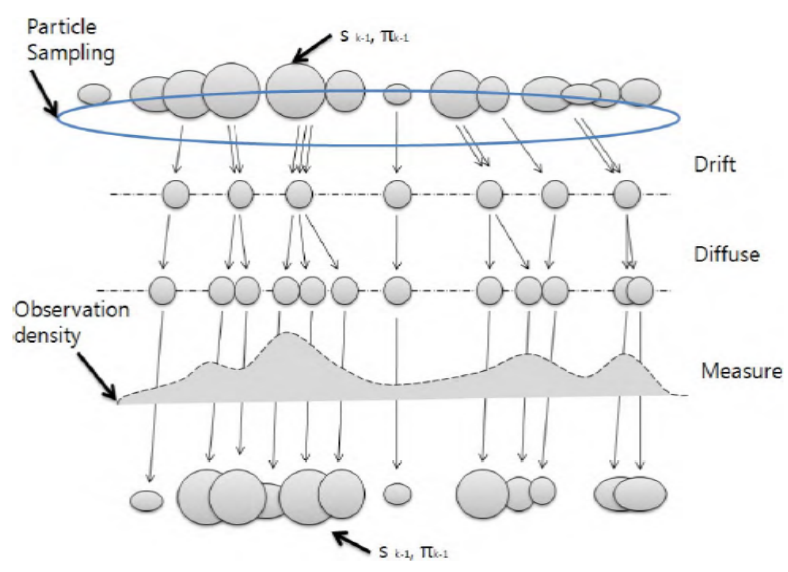

Fig. 3. Once step in the particle filter

\section{Experiment Results}

In this part, one video is adopted to test the performance of our work. This video is available on http://people.csail.mit.edu/ jamesm/index.php

The experiment is implemented using Microsoft Visual studio 2008 on the MS Windows XP SP3 and Intel ${ }^{\circledR}$ Core $^{\mathrm{TM}} 2$ Duo CPU E4700 with 2G RAM.

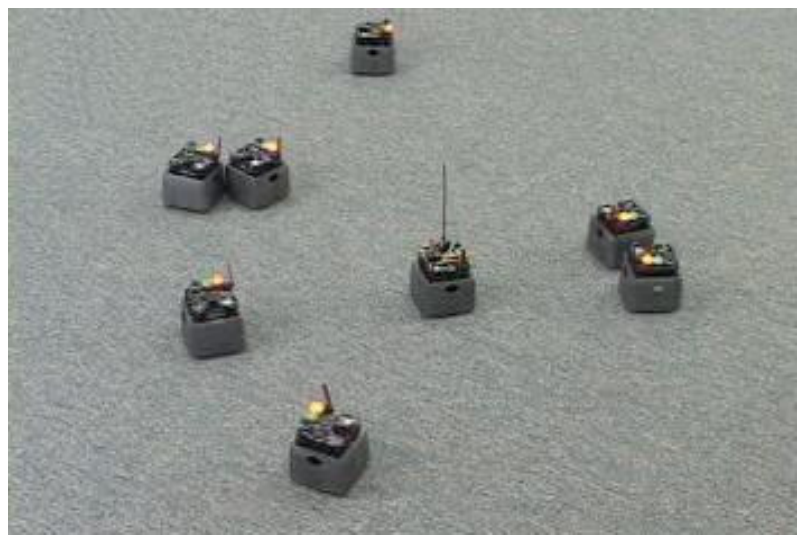

Fig. 4. A typical video frame from our dataset. 


\subsection{Object detection}

We use background subtraction algorithm for object detection. Background subtraction algorithms define the background as parts of a scene at rest. Traditionally these algorithms assume a stationary camera, and identify moving objects by detecting areas in a video that change over time. We compute the difference between the background image and the current frame. Figure 5 shows some frames of the object detection results based on background subtraction algorithm. Background image looks like very clean and smooth However, it is unconstrained environment because it is staining pattern as gaussian noise, not single color.

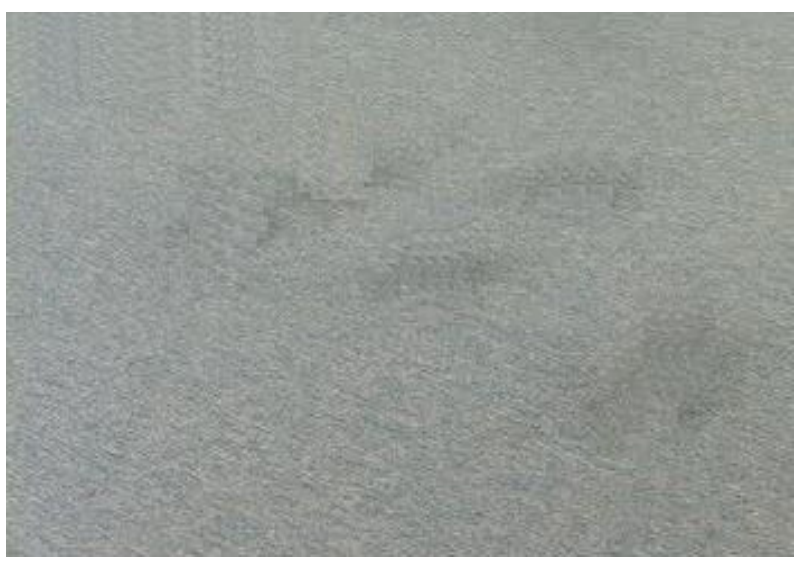

Frame \# 1(background image)

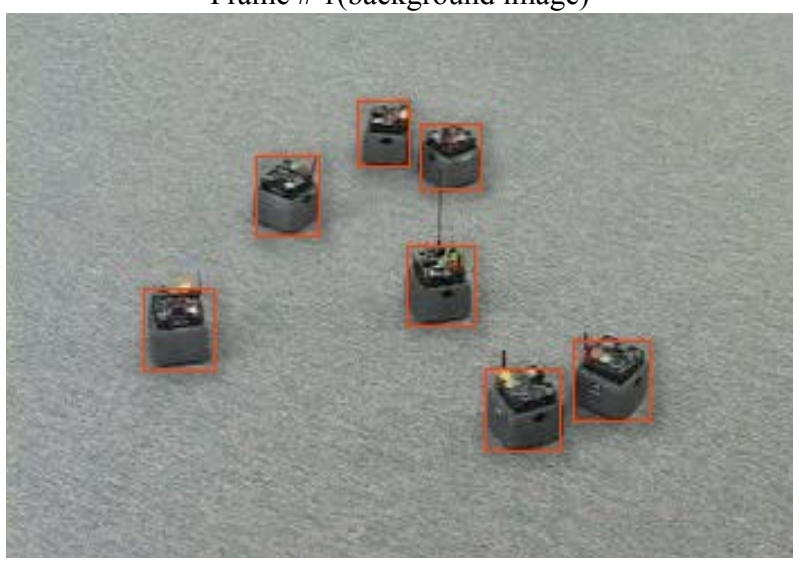

Frame \# 20

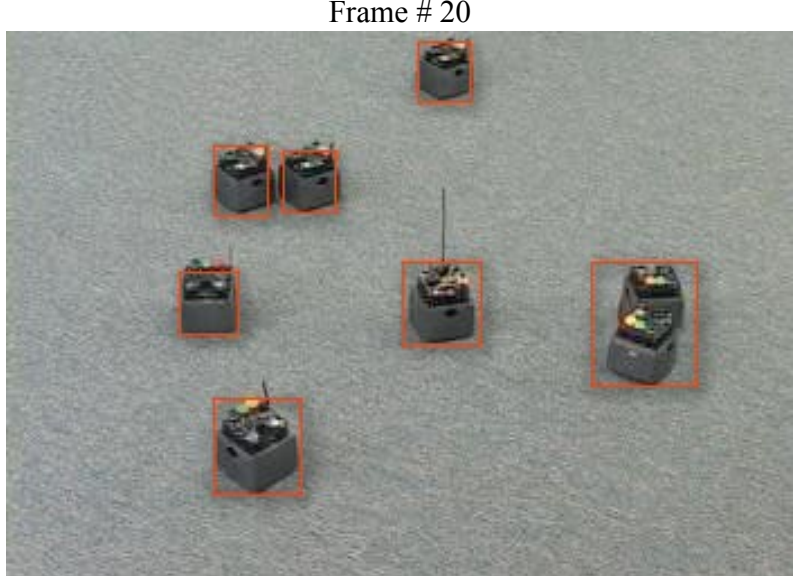

Frame \# 60

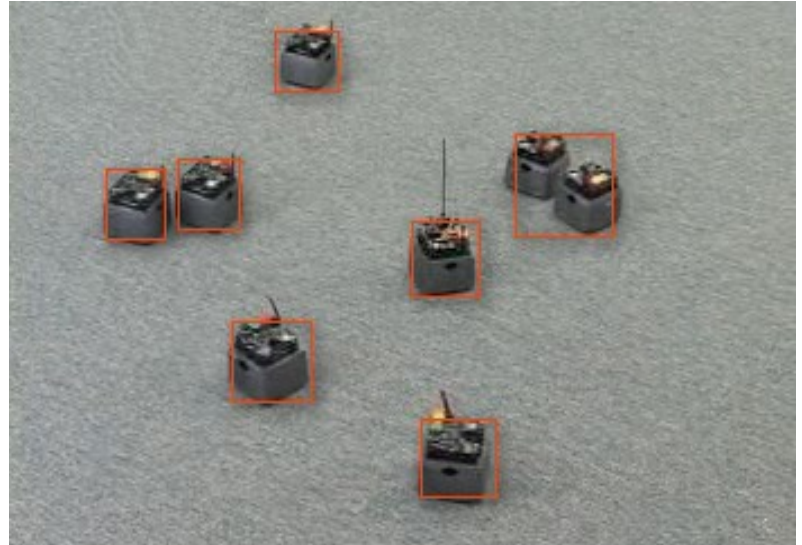

Frame \# 100

Fig. 5. Object detection results

\subsection{Particular object tracking}

When multiple objects were detected by using a background subtraction algorithm, we select particular object. There are difficulties related to particular object tracking, such as erratic object motion, cluttered background and other moving objects. It can't rely only on image processing techniques. Thus we solved these problems using a Particle filter. In this experiment, we resample 50 particles at each frame. Each particle is weighted by its likelihood. Most likely particle represents the object state at time $t$. Before time step $t+1$, particle set is resampled according to particle weights. Figure 6 shows some frames of the particular object tracking results based on particle filter.

\section{Conclusion}

The goal of our experiment is to track particularly selected moving object in an environment of multiple moving objects. We used background subtraction algorithm for object detection. When multiple objects were detected, we selected particular object. And then selected object is tracked. There are problems in tracking a particular object through a sequence of video. It can't rely only on image processing techniques. Thus we solved these problems using particle filter. Particle filter has been proved to be a robust algorithm to deal with the nonlinear, non-Gaussian problems. In this paper, the particle filter provides a robust object tracking framework under ambiguity conditions and estimation accuracy for particular object tracking problems. The previous results show that our method works well. The future works will focus on the particular tracking methods to apply to video captured from a freely moving camera. 


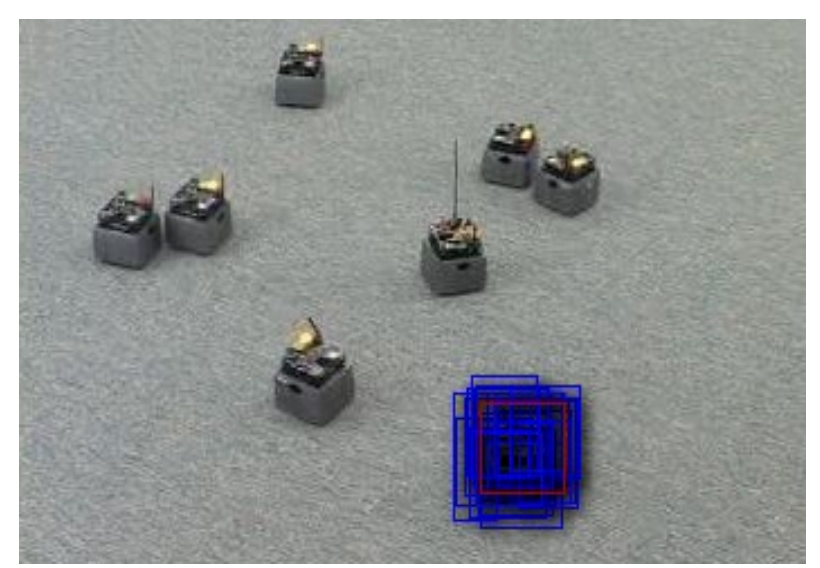

Frame \# 120

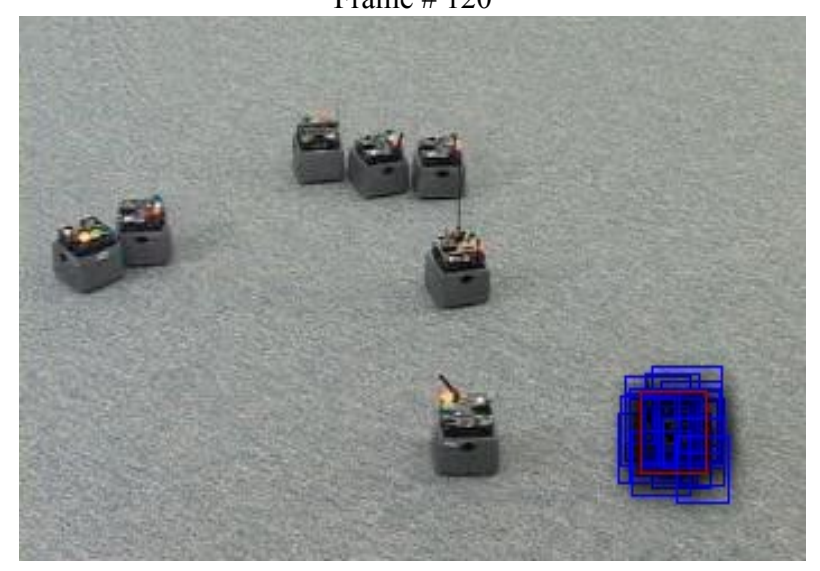

Frame \# 170

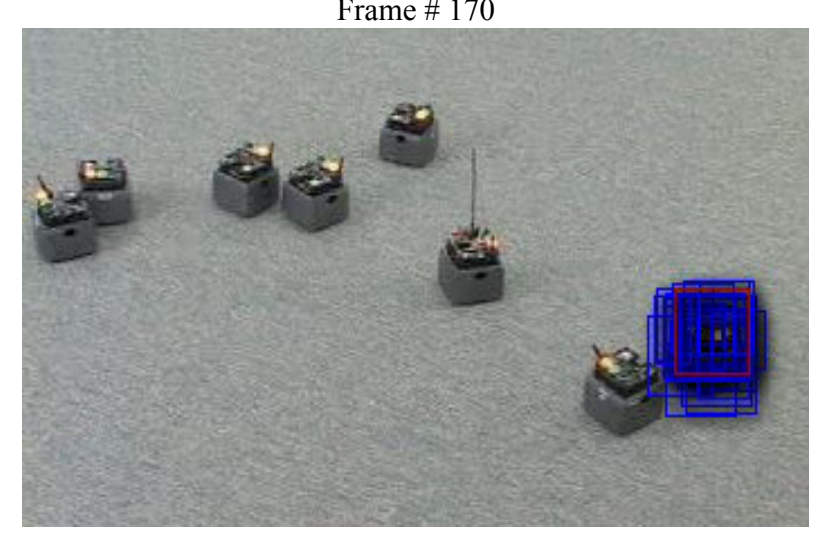

Frame \# 220

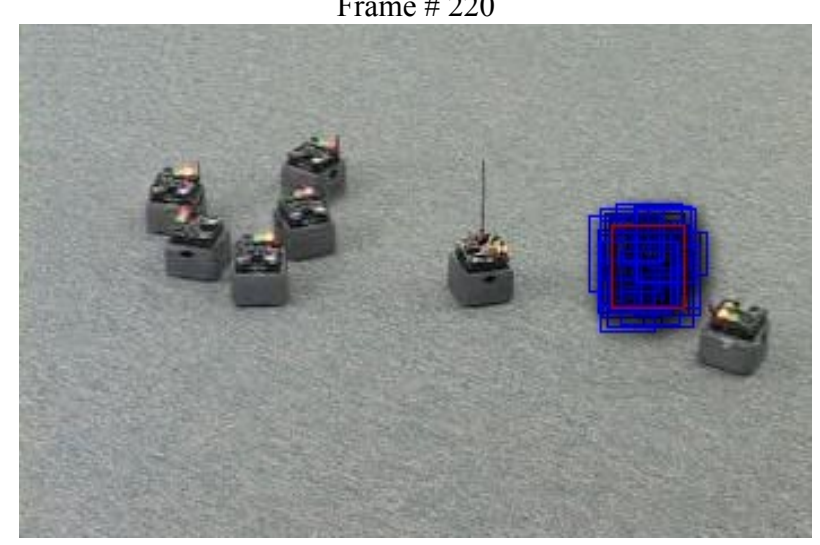

Frame \# 270

Fig. 6. Particular object tracking results

\section{References}

[1] P. Salesmbier, L. Torres, F. Meyer and C. Gu, "Regionbased Video Coding Using Mathematical Morphology," Proc. of the IEEE, vol. 83, no. 6, pp. 843-857, 1995.

[2] Harris C. \& Stennett C. "Rapid - A Video Rate Object Tracker", Proc. British Machine Vision Conference, BMVC-90, Oxford, pp.73-77, 1990.

[3] M. Isard and A. Blake, "Contour Tracking by Stochastic Propagation of Conditional Density," In Proc. European Conf. Computer Vision, pp. 343-356, 1996.

[4] B. Rao, "Data Association Methods for Tracking Systems," In A. Black and A. Yuille, editors, Active Vision, pp. 91-105, MIT, 1992.

[5] Wang, H., et al., "Adaptive object tracking based on an effective appearance filter." IEEE Transactions on Pattern Analysis and Machine Intelligence, vol. 29, no. 9, pp. 16611667, 2007.

[6] Tang, P., et al. Stochastic approach based salient moving object detection using kernel density estimation, Wuhan, China: SPIE, 2007.

[7] Arulampalam M.S., Maskell S., Gordon N., Clapp T., "A Tutorial on Particle Filters for Online Nonlinear/NonGaussian Bayesian Tracking”, IEEE Transactions on Signal Processing, vol. 50, no. 2, pp. 174-188, 2002.

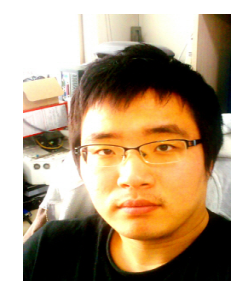

Seung-Min Park

He received the B.S. degree from the Department of Electrical and Electronics Engineering, Chung-Ang University, Seoul, Korea, in 2010. He is currently Master course in the School of Electrical and Electronics Engineering at Chung-Ang University. His research interests include Pattern Recognition, Brain Computer Interface, Evolutionary Computation, etc.

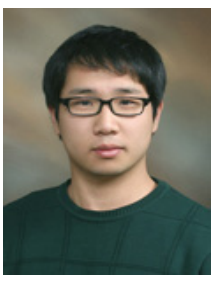

\section{Junheong Park}

He received the B.S. degree from the Department of Electrical and Electronics Engineering, Chung-Ang University, Seoul, Korea, in 2011. He is currently Master course in the School of Electrical and Electronics Engineering at Chung-Ang University. His research interests include Machine Learning, Brain-Computer Interface, Gesture Recognition, etc. 


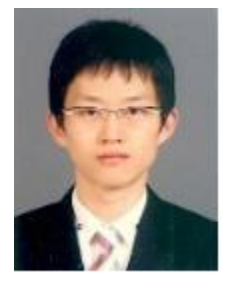

\section{Hyung-Bok Kim}

He received his B.S and M.S. degree in the Department of Electrical and Electronics Engineering from Chung-Ang University, Seoul, Korea, in 2009 and 2011 respectively. His research interests include multi-agent and distributed autonomous robotic system , brain-computer interface, image processing, intelligent robot, machine learning etc.

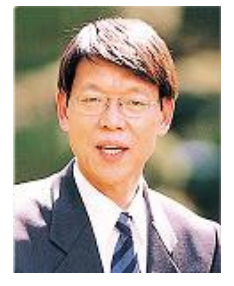

\section{Kwee-Bo Sim}

He received his B.S. and M.S. degrees in the Department of Electronics Engineering from Chung-Ang University, Korea, in 1984 and 1986 respectively, and Ph.D. degree in the Department of Electronics Engineering from The University of Tokyo, Japan, in 1990. Since 1991, he is currently a Professor. His research interests include artificial life, emotion recognition, ubiquitous intelligent robot, intelligent system, computational intelligence, intelligent home and home network, ubiquitous computing and Sense Network, adaptation and machine learning algorithms, neural network, fuzzy system, evolutionary computation, multiagent and distributed autonomous robotic system, artificial immune system, evolvable hardware and embedded system etc. $\mathrm{He}$ is a member of IEEE, SICE, RSJ, KITE, KIEE, KIIS, and ICROS Fellow.

Tel : $+82-2-820-5319$

Fax : +82-2-817-0553

E-mail : kbsim@cau.ac.kr 